\title{
Retrograde signal is the way forward
}

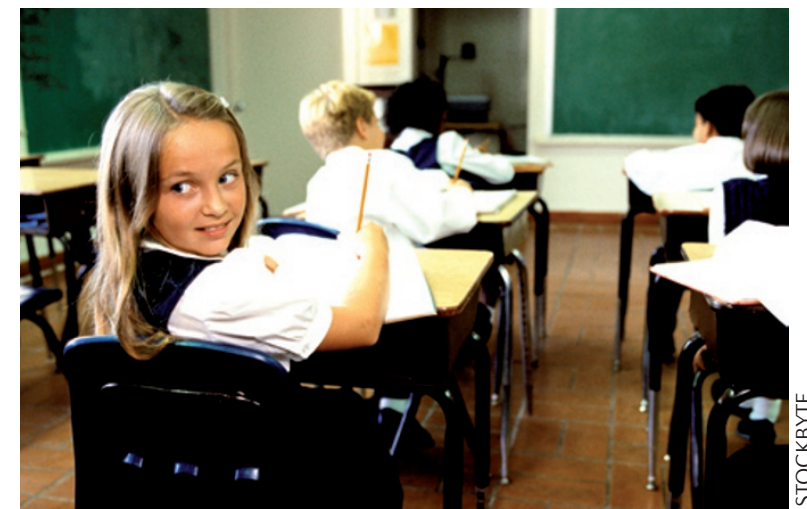

Research on the sensorimotor synapse of the sea slug Aplysia continues to shed light on the molecular mechanisms of synaptic plasticity. A recent report in Current Biology implicates postsynaptic cellular events in long-term facilitation (LTF; a long-lasting form of invertebrate synaptic strengthening that is thought to be important for learning) at this synapse.

The large size of the sea slug's nerve cells and its robust gill- and siphon-withdrawal reflex have enabled researchers to demonstrate that repeated exposure to serotonin, a modulatory neurotransmitter that plays a key part in learning in Aplysia, induces LTF at the sensorimotor synapse. It does so by increasing the expression and release of the neuropeptide sensorin from the presynaptic sensory neuron. It is thought that, by binding to autoreceptors, sensorin activates signal-transduction pathways involving mitogen-activated protein kinases (MAPKs) that lead to long-term changes in synaptic strength. However, the involvement of postsynaptic cellular events was unclear.

Using co-cultures of Aplysia pleural sensory neurons and small siphon-type motor neurons, Glanzman and colleagues show that LTF requires an increase in intracellular $\mathrm{Ca}^{2+}$ levels and protein synthesis in the postsynaptic neuron. Injection of a $\mathrm{Ca}^{2+}$ chelator into the postsynaptic neuron or treatment with membrane-impermeant proteinsynthesis inhibitors prevented serotonin-induced LTF, as measured by changes in excitatory postsynaptic potential (EPSP) amplitude. Moreover, chelating intracellular $\mathrm{Ca}^{2+}$ in the postsynaptic neuron blocked the serotonin-induced increase in sensorin expression in the presynaptic sensory neuron. This finding raises the intriguing possibility that serotonin acts directly on the postsynaptic neuron, through the activation of G-protein-coupled receptors, to trigger not only an increase in $\mathrm{Ca}^{2+}$ but also the release of one or more retrograde signals that mediate the effect of serotonin on presynaptic sensorin expression.

Although postsynaptic cells have been shown to contribute to many forms of synaptic plasticity, this is the first demonstration of the precise postsynaptic cellular events that are required for LTF in Aplysia. Whereas the action of serotonin on presynaptic neurons might produce short-term changes in synaptic plasticity, the authors suggest that the action of serotonin on postsynaptic cells and a subsequent increase in postsynaptic $\mathrm{Ca}^{2+}$ are required for long-term changes in plasticity. Given the similarities with models of long-term potentiation in the mammalian hippocampus involving brain-derived neurotrophic factor and postsynaptic $\mathrm{Ca}^{2+}$, the identification of the retrograde signal(s) that mediates the effect of serotonin on sensorin expression and LTF is eagerly awaited.

Monica Hoyos Flight

ORIGINAL RESEARCH PAPER Cai, D., Chen, S

$\&$ Glanzman, D. L. Postsynaptic regulation of long-term facilitation in Aplysia. Curr. Biol. 18, 920-925 (2008) 